\title{
Peri-tumoral brain edema associated with glioblastoma correlates with tumor recurrence
}

\author{
Xingping Qin ${ }^{1,2 *}$, Rui Liu ${ }^{3 *}$, Farhana Akter ${ }^{4}$ Lingxia Qin ${ }^{5}$, Qiurong Xie ${ }^{6}$, Yanfei Li7 , Haowen Qiao ${ }^{8}$, Wen \\ Zhao $^{8}$, Zhihong Jian ${ }^{1}$, Renzhong Liu ${ }^{\circledR}$, Songlin $\mathrm{Wu}^{9 凶}$ \\ 1. Department of Neurosurgery, Renmin Hospital of Wuhan University, Wuhan, Hubei 430060, China \\ 2. John B. Little Center for Radiation Sciences, Department of Environmental Health, Harvard T.H. Chan School of Public Health, Boston, MA 02115, USA. \\ 3. Department of Physiology, School of Basic Medical Sciences, School of Medicine, Wuhan University, Wuhan, Hubei 430071, China. \\ 4. Faculty of Arts and Sciences, Harvard University, Cambridge, MA 02138, USA. \\ 5. Department of Neurology, Renmin Hospital of Wuhan University, Wuhan, Hubei 430060, China. \\ 6. Department of Gynecology and Obstetrics, Renmin Hospital of Wuhan University, Wuhan, Hubei 430060, China. \\ 7. Department of Orthopedics, The First Affiliated Hospital of Jinan University, Guangzhou 510630, China. \\ 8. Department of Biomedical Engineering, Wuhan University School of Basic Medical Sciences, Wuhan, Hubei 430071, China. \\ 9. Department of Geriatrics, Renmin Hospital of Wuhan University, Wuhan, Hubei 430060, China. \\ * These authors contributed equally to this work.
}

$\triangle$ Corresponding authors: Renzhong Liu (liurenzhong@whu.edu.cn) \& Songlin Wu (wusonglin@whu.edu.cn), Department of Neurosurgery, Renmin Hospital of Wuhan University, 99 Zhang Zhidong Rd, Wuhan, Hubei 430060, China.

(C) The author(s). This is an open access article distributed under the terms of the Creative Commons Attribution License (https://creativecommons.org/licenses/by/4.0/). See http://ivyspring.com/terms for full terms and conditions.

Received: 2020.09.12; Accepted: 2020.12.29; Published: 2021.02.05

\begin{abstract}
Glioblastoma is the most common malignant tumor of the brain. Despite advances in treatment, the prognosis for the condition has remained poor. Glioblastoma is often associated with peritumoral brain edema (PTBE), which can result in increased intracranial pressure and devastating neurological sequelae if left untreated. Surgery is the main treatment for glioblastoma, however current international surgical guidelines do not specify whether glioblastoma-induced PTBE tissue should be resected. In this study, we analyzed treatment outcomes of PTBE using surgical resection. We performed a retrospective analysis of 255 cases of glioblastoma between 2014 and 2016, and found that a significant proportion of patients had a degree of PTBE. We found that surgical resection led to reduction in midline shift that had resulted from edema, however, postoperative complications and KPS scores were not significantly different in the two conditions. We also observed a delay in glioblastoma recurrence in patients undergoing PTBE tissue resection vs patients without resection of PTBE tissue. Interestingly, there was an abnormal expression of tumor associated genes in PTBE, which has not been previously been found. Taken together, this study indicates that glioblastoma-induced PTBE should be investigated further particularly as the tumor microenvironment is a known therapeutic target and therefore interactions between the microenvironment and PTBE should be explored. This study also highlights the importance of resection of PTBE tissue to not only reduce the mechanical obstruction associated with edema but also to delay recurrence of glioblastoma.
\end{abstract}

Key words: Glioblastoma; Peritumoral brain edema; Recurrence; Tumor resection.

\section{Introduction}

Gliomas are a heterogenous group of brain tumors. The World Health Organization (WHO) classification system classifies gliomas from grade 1 to grade 4. Grade 1 and 2 gliomas are referred to as low-grade gliomas (LGG), while grade 3 and 4 gliomas are referred to as high-grade gliomas (HGG) [1]. Glioblastoma (GBM) is the most malignant form and is usually treated with surgical resection. However, the prognosis for GBM remains poor, with an average survival time of 14 months post treatment [2]. The preoperative evaluation of GBMs relies on magnetic resonance imaging (MRI), which has the highest sensitivity to tumors [3]. The standard surgical approach is to maximally resect tumors 
whilst preserving neurological function. However, GBM is associated with high relapse rates and therefore has an unfavorable prognosis.

Tumors are often accompanied by abnormal expression of tumor-associated genes. The AKT gene, also known as protein kinase B plays an important role in cell survival and activation of AKT promotes cell proliferation $[4,5,6]$. The extracellular signal-regulated kinase (ERK) signaling pathway is at the core of signaling networks that regulate cell growth, development, and division $[7,8]$. There are several tumor suppressor genes that can be affected in tumorigenesis. Phosphatase and tension homologue deleted on chromosome 10 (PTEN) and P53 are tumor suppressors that are frequently disrupted during carcinogenesis. Their expression is maintained in normal cells but are significantly down-regulated during carcinogenesis $[9,10]$.

Previous studies have indicated that the metabolic circuit of glioma cells changes during carcinogenesis, with evidence of an increase in glucose uptake and lactic acid production regardless of the availability of oxygen [11]. We call this phenomenon aerobic glycolysis or Warburg effect, and this is closely related to tumor growth [12]. Direct regulators of the Warburg effect include Pyruvate kinase M (PKM2), phosphoglycerate kinase 1 (PGK1), lactate dehydrogenase A (LDHA), c-Myc oncogene and Glucose transporter 1 (GLUT1) [13,14]. An increase in PKM2 expression level in tumors further promotes LDHA, c-Myc and GLUT1 expression [15]. PGK1 promotes the Warburg effect in tumors by suppressing tricarboxylic acid cycle (TCA) through mitochondrial translocation [13,14,16,17] (Fig. 1). Peritumoral brain edema (PTBE) is commonly found in GBM and is thought to be associated with poorer prognosis [18] and increased morbidity such as cognition deficits [19]. PTBE can also be found in metastatic brain tumors and benign tumors such as meningiomas [20].

There is controversy regarding whether PTBE associated with GBM should be resected. In this study we demonstrate that surgical resection of PTBE tissue does not confer a greater post-operative risk compared to patients who do not receive surgical resection. Most interestingly, we demonstrate that GBM associated PTBE is associated with an upregulation of tumor-associated genes, which may be involved in the pathogenesis of GBM formation and evolution and should be explored further.

\section{Materials and Methods}

\section{Peritumoral brain edema (PTBE)}

The maximum diameter of the tumor was measured on $\mathrm{T} 1$ weighted images (T1WI), and the maximum diameter of the edema band was measured axially on the T2-weighted images (T2WI) or Fluid-attenuated inversion recovery (FLAIR) sequence images. PTBE was evaluated for the maximum diameter of the edema and was classified into 3 grades: Level 0, no edema; Level 1, maximum diameter less than 1cm; Level 2, maximum diameter $>1 \mathrm{~cm}$. Axial, coronal and sagittal MRI scans were reviewed in all cases and the maximum extent of PTBE was measured using these scans.

\section{Neurosurgery}

We divided patients with PTBE into two groups: one group received surgical resection of the tumor and PTBE tissue and the other group received surgical resection of tumor only. In this study, 50 males (51\%) and 35 females (53.8\%) received surgical resection of PTBE tissue in addition to tumor resection, while 48 males (49\%) and 30 females (46.2\%) received surgical excision of tumor only. We also categorized patients according to age as young ( $<60$ years) and elderly ( $\geq 60$ years). 53 patients $(55.8 \%)$ in the younger group and 32 patients $(47.1 \%)$ in the elderly group received both PTBE and tumor resection, whilst 42 (44.2\%) young patients and $36(52.9 \%)$ elderly patients received surgical excision of the tumor only.

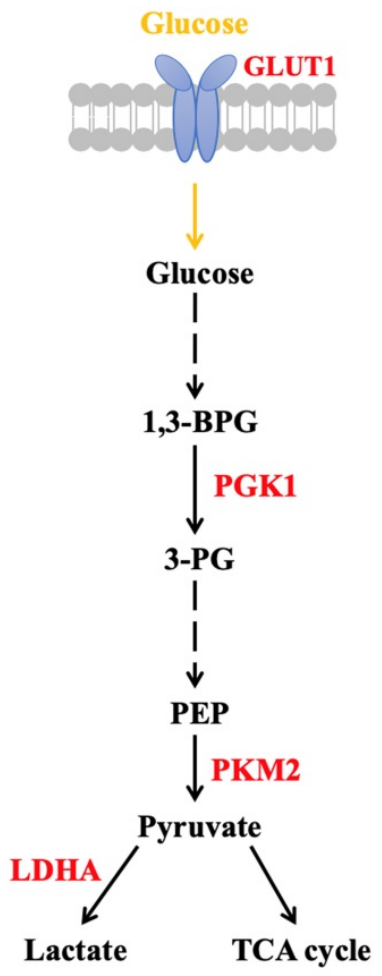

Figure 1. Warburg effect and tumor growth 
We used T2WI and T2-FLAIR MRI images and diffusion tensor imaging (DTI) as a reference during surgery. All operations were conducted with a microscope. For the tumor only group, we resected the tumor completely and ensured that the surrounding normal brain tissue was preserved. We resected the PTBE tissue according to T2W1 and T2-FLAIR images, aiming to preserve as much of the eloquent brain areas as possible without damaging the nerve fiber tracts. Patients were subjected to intraoperative neurophysiological monitoring under awake conditions to identify any neurological impairment. Some patients underwent chemotherapy postoperatively, while some underwent radiotherapy, and some received combination of chemotherapy and radiotherapy. All cases were categorized according to their pathology, in the "tumor only" group, 35 patients $(44.80 \%)$ were classified as of the MGMT methylated type, 70 patients $(89.70 \%)$ as IDH1 mutated, 40 patients $(51.30 \%)$ as EGFR amplified, 42 patients $(53.80 \%)$ as PI3K activated, 4 patients $(5.12 \%)$ with $1 \mathrm{p} / 19 \mathrm{q}$ codeletion, 19 patients $(24.35 \%)$ with P53 mutations, and 42 patients (53.85\%) with PTEN mutations. In the "tumor only + PTBE" group, there are 38 patients $(44.71 \%)$ who had MGMT methylation, 77 patients $(90.59 \%)$ with IDH1 mutation, 45 patients $(52.94 \%)$ with EGFR amplification, 43 patients (50.59\%) with PI3K activation, 6 patients $(7.06 \%)$ with 1p/19q codeletion, 22 patients $(25.88 \%)$ with P53 mutation, and 48 patients $(56.47 \%)$ with PTEN mutation.

\section{Human PTBE brain tissues}

Human PTBE brain tissues were obtained at the time of surgery from Department of Neurosurgery in Renmin Hospital of Wuhan University, and histological diagnosis was confirmed independently by three neuropathologists. The procurement of tissue usage and all image data for the study was obtained with written patient-informed consent and approved by the Institutional Ethics Committee Faculty of Medicine at Renmin Hospital of Wuhan University (approval number: 2012LKSZ(010)H).

\section{Normal human brain tissues}

Normal brain tissue was obtained from donors who had no known diseases or pathology at post-mortem via the Chinese Brain Bank Center (CBBC) through a human body donation program, organized by the Wuhan Red Cross Society. Specific permission for the use of autopsy results and medical data for research purposes were obtained either from the donors themselves or from their relatives, and was also approved by the Biomedical Research Ethics Committee of South-Central University for
Nationalities (approval number: 2017-SCUEC-MEC004).

\section{KPS}

The patients were assessed by Karnofsky performance scale (KPS). This is an assessment tool to identify functional impairment when comparing effectiveness of different therapies. The score is calculated from 0 to 100, with 0 representing death and 100 representing no signs or symptoms of disease. A score $\geq 70$ signifies that one is able to care for themselves independently.

\section{Midline shift}

Midline shift is a measure of the brain past its center line and indicates a mass effect from the presence lesions such as a tumor or edema. Midline shift is a common characteristic of GBM. The presence of midline shift is considered as an independent prognostic factor influencing survival among GBM patients [21]. In this study, we measured the distance between septum pellucidum from the midline using MRI and is classified into 2 groups: $<1 \mathrm{~cm}$ and $>1 \mathrm{~cm}$.

\section{Western blotting analysis}

Western blotting was performed using a standard protocol. Briefly, the polyvinylidene difluoride (PVDF) membrane by Millipore (USA) was used to incubate with the first antibody against AKT (Mouse, 1:1000), phospho-AKT (Ser473) (Rabbit, 1:1000), ERK 1/2 (Rabbit, 1:2000), phospho-ERK 1/2 $\left(\mathrm{Thr}^{202} / \mathrm{Tyr}^{204}\right.$ ) (Rabbit, 1:2000), PTEN (Rabbit, 1:1000), P53 (Mouse, 1:1000), PKM2 (Rabbit, 1:1000), LDHA (Rabbit, 1:1000), c-Myc (Rabbit, 1:1000), Actin (Rabbit, 1:2000) (\#8457) from Cell Signaling Technology (MA, USA), PGK1 (Rabbit, 1:2000), GLUT1 (Rabbit, 1:1000), GAPDH (Mouse, 1:5000) from Proteintech Group (USA), NLGN3 (Mouse, 1:500) (sc-137052) that was from Santa Cruz Biotechnology. The primary antibody was labeled with the secondary antibody and the protein bands were imaged using SuperSignal West Femto Maximum Sensitivity Substrate (Pierce, Rockford, IL, USA). Blot images were obtained directly from the PVDF membrane using an EC3 imaging system (UVP, LLC, Uplant, USA). Western blot data was quantified using Image J Pro Plus 6.0 software.

\section{Statistics}

All data are expressed as mean \pm SE. Newman-Keuls tests were used for post-hoc comparisons when appropriate. Chi-Square tests were used to compare KPS scores and midline shift between resected and non-resected cases. The Fisher Exact test was used to compare recurrence rates following resection of PTBE vs patients who did not 
receive resection. $P<0.05$ was considered statistically significant.

\section{Results}

\section{Glioblastoma induces peritumoral brain edema}

A retrospective study was performed on 255 GBM patients from 2014 to 2016, who underwent tumor resection. Among them, 207 patients were followed up for more than 1 year and 48 patients were not followed up due to death or unknown reasons. Demographic characteristics of patients are specified in Table 1. Data shows that the median age in this cohort is 52 years. In the 207 patients who were followed up, 55.07\% (114/207) were men and 44.93\% $(93 / 207)$ were women. The primary site of GBM occurrence was in the frontal lobe (84 cases), followed by the temporal lobe (67 cases), 35 cases in the parietal lobe, and 21 cases in the occipital lobe. PTBE was graded into 3 grades as follows: Level 0 (no edema); Level 1 (maximum diameter of edema less than $1 \mathrm{~cm}$ ); Level 2 (maximum diameter of edema $>1 \mathrm{~cm}$ ). We found that brain edema was present in $78.74 \%$ $(163 / 207)$ of the cases of GBM) (Fig. 2). There were 92 cases graded as level 0, 36 cases graded as level 1 and 127 cases graded as level 2.

Table 1. Demographic characteristics of patients

\begin{tabular}{ll}
\hline GBM (patients) & $\mathbf{2 5 5}$ \\
\hline Age (years) & 52 \\
Median & $40-62$ \\
Range & 207 \\
Gender (patients) & 114 \\
Male & 93 \\
Female & 207 \\
Primary site of GBM (patients) & 84 \\
Frontal lobe & 67 \\
Temporal lobe & 35 \\
Parietal lobe & 21 \\
Occipital lobe & 163 \\
Edema on primary GBM (patients) & 36 \\
Level 1 & 127 \\
Level 2 & \\
\hline
\end{tabular}

Table 2. Postoperative symptoms

\begin{tabular}{|c|c|c|c|c|c|c|c|}
\hline \multicolumn{8}{|c|}{ PTBE patients (163) } \\
\hline & \multirow[t]{2}{*}{ Pre-operation } & \multicolumn{3}{|c|}{$\begin{array}{l}\text { PTBE resection } \\
\text { (85) }\end{array}$} & \multicolumn{3}{|c|}{$\begin{array}{l}\text { PTBE no resection } \\
\text { (78) }\end{array}$} \\
\hline & & $1 \mathrm{M}$ & $3 \mathrm{M}$ & $6 \mathrm{M}$ & $1 \mathrm{M}$ & $3 \mathrm{M}$ & $6 \mathrm{M}$ \\
\hline \multicolumn{8}{|c|}{ Neurologic impairment } \\
\hline Headache & 78 & 36 & 42 & 31 & 32 & 34 & 35 \\
\hline Vomiting & 23 & 7 & 10 & 15 & 10 & 12 & 19 \\
\hline Paresthesia & 43 & 26 & 29 & 33 & 28 & 30 & 34 \\
\hline Hemiplegia & 56 & 26 & 33 & 39 & 30 & 36 & 38 \\
\hline $\begin{array}{l}\text { Visual } \\
\text { impairment }\end{array}$ & 21 & 6 & 9 & 10 & 11 & 11 & 12 \\
\hline Aphasia & 14 & 8 & 8 & 11 & 10 & 8 & 12 \\
\hline Seizures & 12 & 4 & 3 & 5 & 6 & 4 & 5 \\
\hline
\end{tabular}

Management of PTBE is based on the clinical condition of the patient

All patients received total excision of their tumors. However, the decision to surgically resect PTBE tissue based on the extent of PTBE. In total, 85 patients received surgical resection of PTBE tissue in addition to tumor resection and 78 patients received surgical excision of their tumor only (Table 2). In one example case of a patient with right frontal lobe tumor in Fig. 3a, there was little evidence of PTBE and therefore we decided to resect the tumor only. In Fig. $3 \mathrm{~b}$, the patient presented with a right frontal lobe tumor, with contrast enhancement in T1WI-enhanced images and PTBE grading of level 1 . We therefore resected both tumor and PTBE tissue. In Fig. 3c, the patient presented with a tumor in the left frontal lobe, with contrast enhancement and PTBE level 1. This patient received surgical resection of tumor and PTBE tissue. In Fig. 3d, the patient presented with a tumor in the right frontal lobe, with contrast enhancement and PTBE level 2; this patient therefore had surgical excision and removal of PTBE tissue.

\section{Postoperative complications and KPS scores do not show significant differences in patients with PTBE excision or no resection}

Neurological complications following surgery include headache, vomiting, paresthesia, hemiplegia, visual impairment, aphasia and seizures. In Table 3, we recorded in detail the number of patients with corresponding postoperative complications at 1 month, 3 months, and 6 months after surgery.

In patients with headache, PTBE tissue resection accounted for $42.75 \%$ of cases, compared to $43.16 \%$ for cases without resection. In patients with vomiting, PTBE tissue resection accounted for $12.55 \%$ cases vs $17.52 \%$ patients who did not receive resection. In patients with paresthesia, PTBE resection accounted for $34.51 \%$ cases, vs $39.32 \%$ for patients who did not receive resection. In patients with hemiplegia, PTBE resection accounted for $38.43 \%$, vs $44.4 \%$ of patients without resection. In patients with visual impairment, PTBE resection accounted for $9.8 \%$ cases vs $14.53 \%$ for those without resection. In patients with aphasia, PTBE resection accounted for $10.59 \%$, vs $12.82 \%$ in patients without resection. In patients with seizures, PTBE resection accounted for $5.13 \%$ cases, vs $6.41 \%$ in patients without resection. Together, we did not observe a significant difference in the postoperative complications between resected cases vs those that did not resection of PTBE. 


\section{Preoperation}

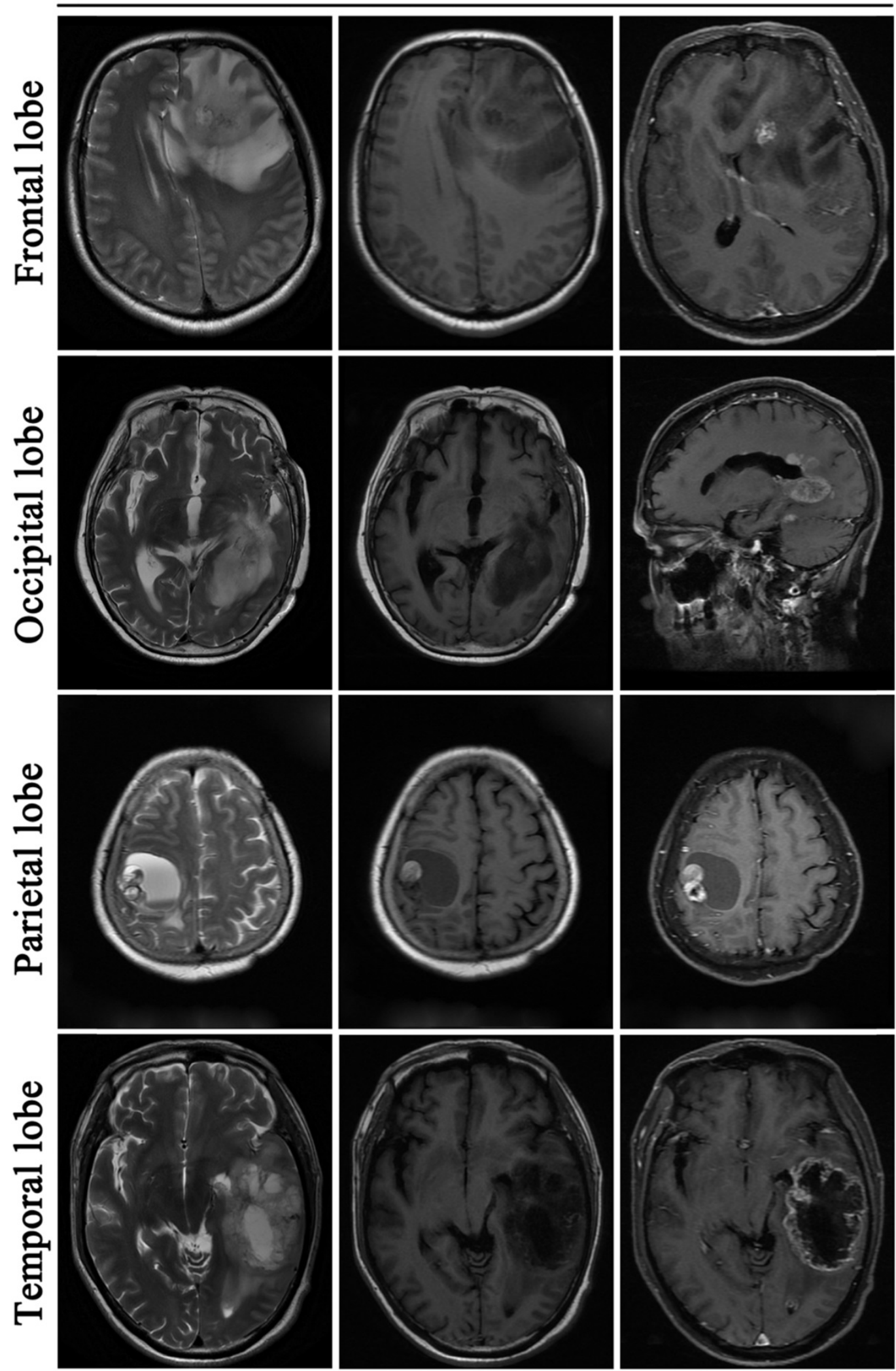

Figure 2. Glioblastoma induces peritumoral brain edema. All the tumors are located in the cortex: Frontal lobe, Occipital lobe, Parietal lobe, Temporal lobe. Before the operative, these patients are accompanied by peritumoral brain edema. 


\section{Preoperation}

a
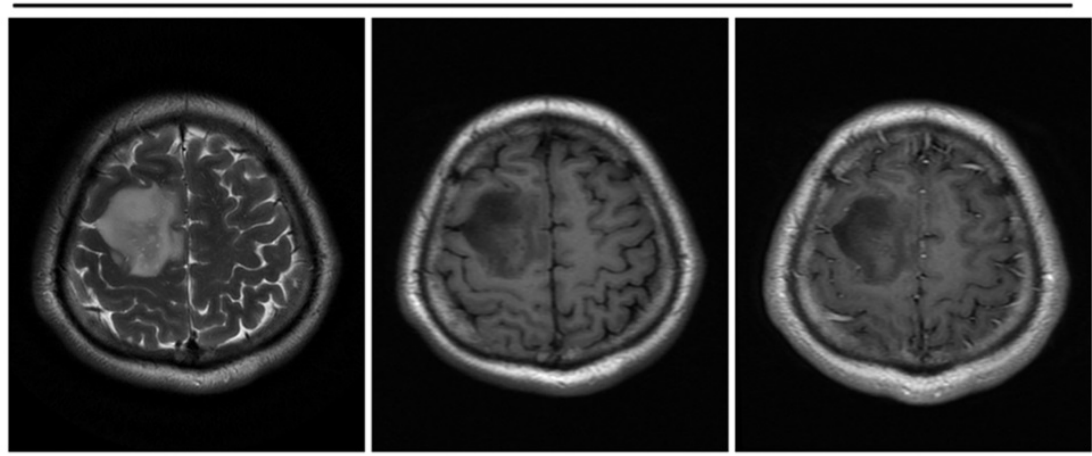

\section{Postoperation}

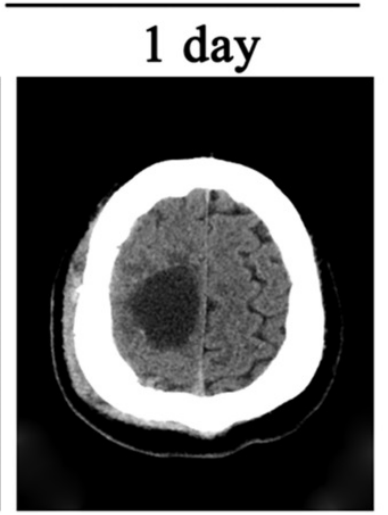

b
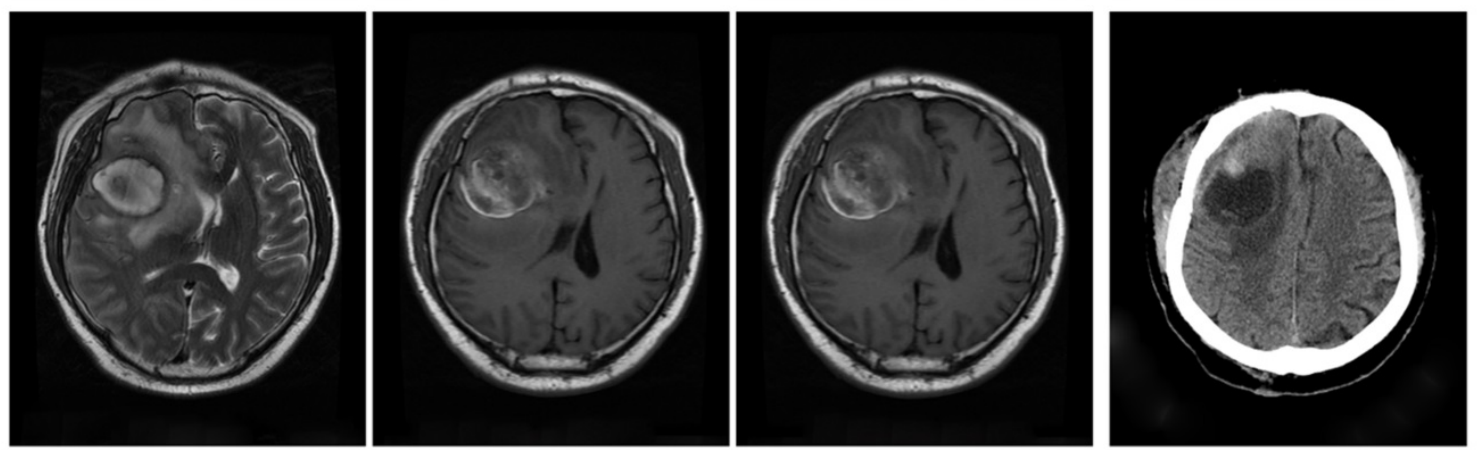

c
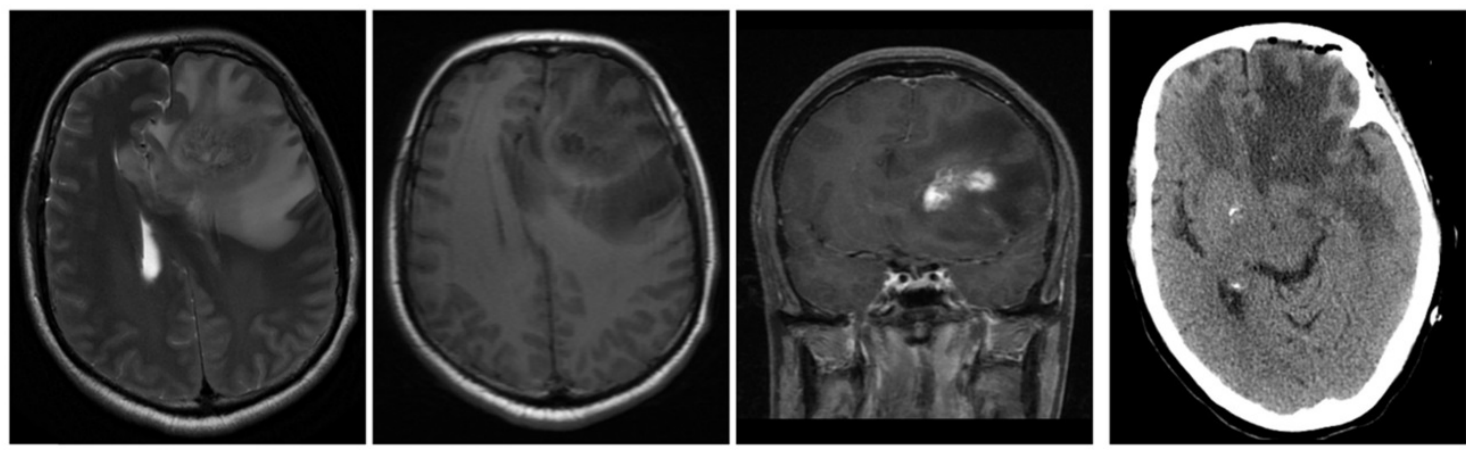

d
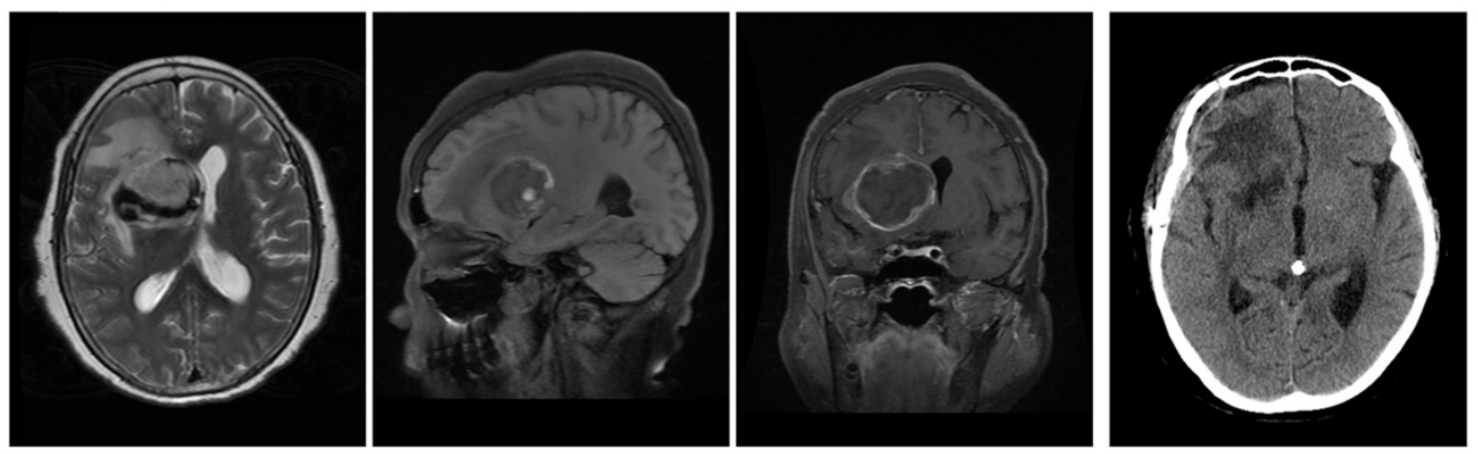

Figure 3. Surgical resection of PTBE is clinically based on the condition of patients. All the patients are accompanied by peritumoral brain edema before operative. $a$ and $c$, patients only take a resection of the tumor. $b$ and $d$, patients remove the tumor and give an excision with PTBE.

We performed KPS scores on all GBM patients after tumor resection to evaluate the prognosis. In 163 GBM patients with PTBE, KPS scores were above 70 points $(\geq 70,91.41 \% ;<70,8.59 \%)$. In all patients with a KPS score greater than 70 , PTBE tissue resection accounted for $92.55 \%$, vs $94.02 \%$ in patients who did not receive resection of PTBE tissue. In all patients with a KPS score less than 70, PTBE tissue resection accounted for $7.45 \%$, vs $5.98 \%$ in those without resection. These data suggest that patients do not show significant differences in KPS scores after resection of PTBE tissue compared to patients who did not receive resection. 
Table 3. Postoperative complications

\begin{tabular}{|c|c|c|c|c|c|c|c|c|c|c|}
\hline & \multirow[b]{2}{*}{ Pre-operative } & \multicolumn{3}{|l|}{$1 \mathrm{M}$} & \multicolumn{3}{|l|}{$3 \mathrm{M}$} & \multicolumn{3}{|l|}{$6 \mathrm{M}$} \\
\hline & & $\begin{array}{l}\text { PTBE resection } \\
\text { (85) }\end{array}$ & $\begin{array}{l}\text { PTBE no resection } \\
\text { (78) }\end{array}$ & $\begin{array}{l}\mathrm{P} \\
\text { value }\end{array}$ & $\begin{array}{l}\text { PTBE resection } \\
\text { (85) }\end{array}$ & $\begin{array}{l}\text { PTBE no resection } \\
\text { (78) }\end{array}$ & $\begin{array}{l}\mathrm{P} \\
\text { value }\end{array}$ & $\begin{array}{l}\text { PTBE resection } \\
\text { (85) }\end{array}$ & $\begin{array}{l}\text { PTBE no resection } \\
\text { (78) }\end{array}$ & $\begin{array}{l}\mathrm{P} \\
\text { value }\end{array}$ \\
\hline \multicolumn{11}{|c|}{ KPS score } \\
\hline$\geq 70$ & 149 & 81 & 76 & 0.468 & 76 & 73 & 0.341 & 79 & 71 & 0.652 \\
\hline$<70$ & 14 & 4 & 2 & 0.468 & 9 & 5 & 0.341 & 6 & 7 & 0.652 \\
\hline \multicolumn{11}{|c|}{ Midline shift } \\
\hline$<1 \mathrm{~cm}$ & 63 & 76 & 60 & 0.032 & 58 & 42 & 0.059 & 40 & 23 & 0.214 \\
\hline$\geq 1 \mathrm{~cm}$ & 100 & 9 & 18 & 0.032 & 27 & 36 & 0.059 & 45 & 55 & 0.214 \\
\hline \multicolumn{11}{|c|}{ Recurrence } \\
\hline & & 0 & 2 & 0.227 & 10 & 28 & 0.004 & 74 & 48 & 0.0002 \\
\hline
\end{tabular}

\section{PTBE resection eases midline shift in GBM patients}

Midline shift is a relatively common phenomenon in GBM patients. In Table 3, statistical analysis revealed significantly improved midline shift in 163 patients undergoing PTBE tissue resection. In 163 GBM patients with PTBE, midline shift pre-operatively was greater than $1 \mathrm{~cm}$ in the majority of the cases $(>1 \mathrm{~cm}, 61.35 \% ;<1 \mathrm{~cm}, 38.65 \%)$. PTBE tissue resection accounted for $68.24 \%$ of cases with midline shift less than $1 \mathrm{~cm}$ post-operatively, vs $53.42 \%$ in patients who did not receive resection, In all patients with a midline shift $>1 \mathrm{~cm}$, PTBE resection accounted for $31.76 \%$.vs. $46.58 \%$ in patients who did not receive resection. This suggests that patients show significant differences in midline shift after resection of PTBE tissue compared to those who did not receive resection at 1 month and 6 months after surgery, however, there was no significant difference at 3 months after surgery.

\section{GBM recurrence in patients undergoing $P T B E$ resection is slower than that of PTBE without resection}

GBM is the most aggressive tumor in the brain with high rates of recurrence even following complete surgical resection. In this study, 207 patients underwent total tumor resection, however they all relapsed within 12 months after operation. In 163 GBM patients with PTBE, the recurrence time of patients who also received resection of PTBE tissue was delayed compared to patients without PTBE tissue (Table 3 and Fig. 4). In patients with both tumor and PTBE tissue resection, there was no recurrence at 1 month's follow-up (0\%); 10 patients relapsed at 3 months' follow-up (11.77\%), and 74 patients relapsed at 6 months' follow-up $(87.06 \%)$. In the group with surgical excision of tumor only, 2 patients relapsed at 1 month's follow-up (2.56\%), 28 patients relapsed at 3 months' follow-up (35.90\%), and 48 patients relapsed at 6 months' follow-up (61.54\%).

\section{Abnormal expression of tumor-associated proteins in PTBE region}

We collected tissues from patients with PTBE and normal human brain tissues. In Fig. 5a we show that levels of phosphorylated AKT (p-AKT) and the phosphorylated of ERK 1/2 (p-ERK 1/2) were higher in brain tissues with PTBE compared to control. We also tested the levels of tumor suppressor genes PTEN and P53, and found reduced expression of these in PTBE brain tissues compared to normal brain tissues (Fig. 5b). Previous studies have demonstrated that PKM2, PGK1, LDHA, c-Myc and GLUT1 plays a crucial role in tumorigenesis. Western blot results showed that PKM2, PGK1, LDHA, c-Myc and GLUT1 expression in PTBE brain tissue were up-regulated compared to normal brain tissue (Fig. 5c). In addition, recent studies have shown that NLGN3 plays an important role in tumor growth [4,22]. Our recent report shows that NLGN3 levels are associated with postoperative recurrence of gliomas [5], western blotting analysis of NLGN3 showed that the level of NLGN3 was higher in the PTBE region compared to normal brain tissue (Fig. $5 \mathrm{~d}$ ). The results suggest that increased expression of tumor-associated proteins in the PTBE region may be associated with relatively rapid recurrence of tumors.

\section{Discussion}

Glioblastoma is the most aggressive tumor in the brain [23]. Surgery followed by radiotherapy and chemotherapy is the most common modality of treatment. However, the prognosis of the condition remains poor, with a survival time of patients only 14 months [5]. We conducted a retrospective study of 255 GBM patients from 2014 to 2016, of which 207 patients were followed up for more than 1 year. In these patients, the primary location of GBM were the frontal lobe in 84 cases, the temporal lobe in 67 cases, the parietal lobe in 35 cases, and the occipital lobe in 21 cases. We found that in all these cases, there was a degree of PTBE. Despite being commonly reported in the literature, the pathogenesis underlying GBM associated PTBE is unclear. 
$\mathbf{a}$

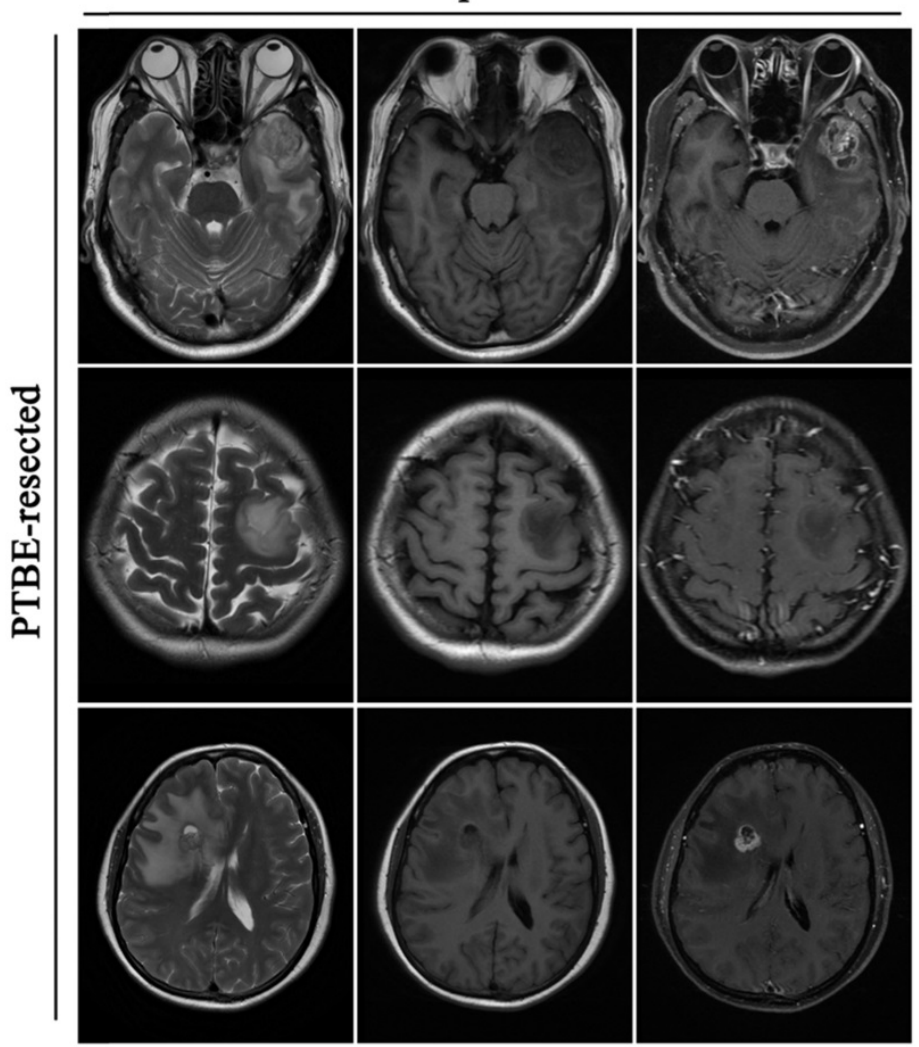

b

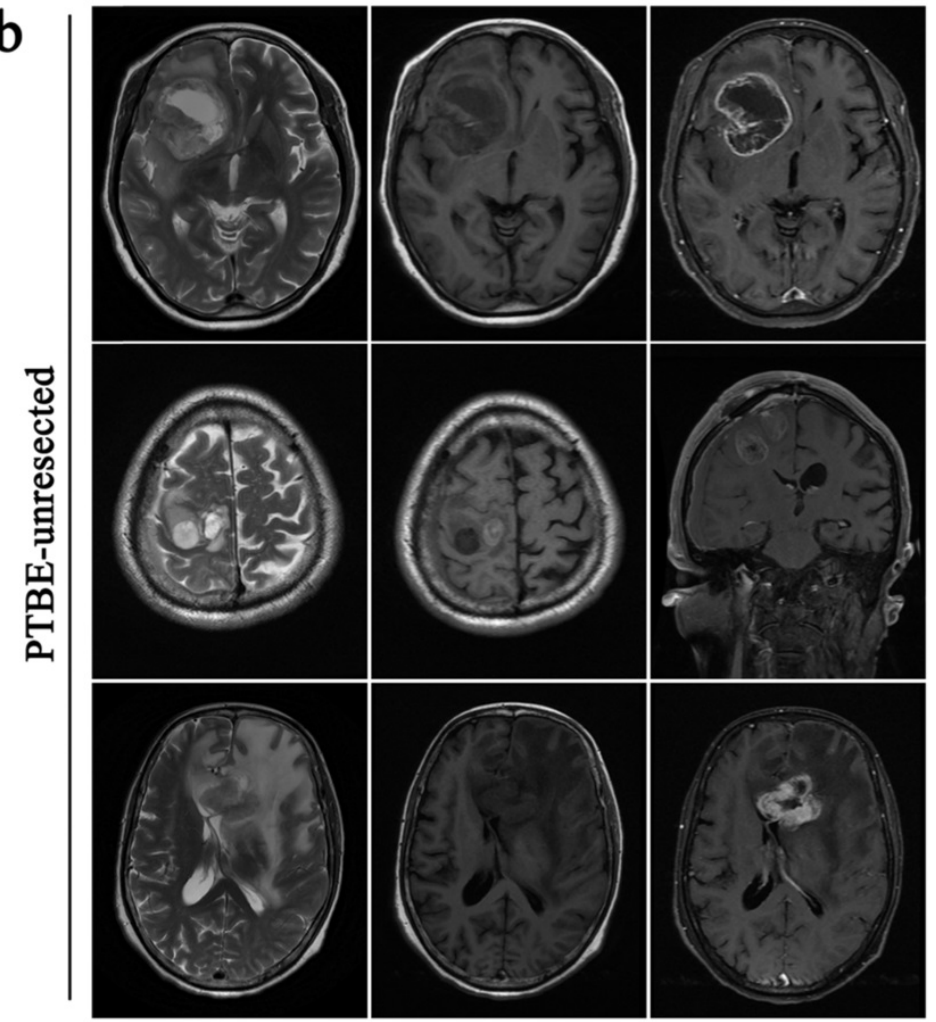

Postoperation
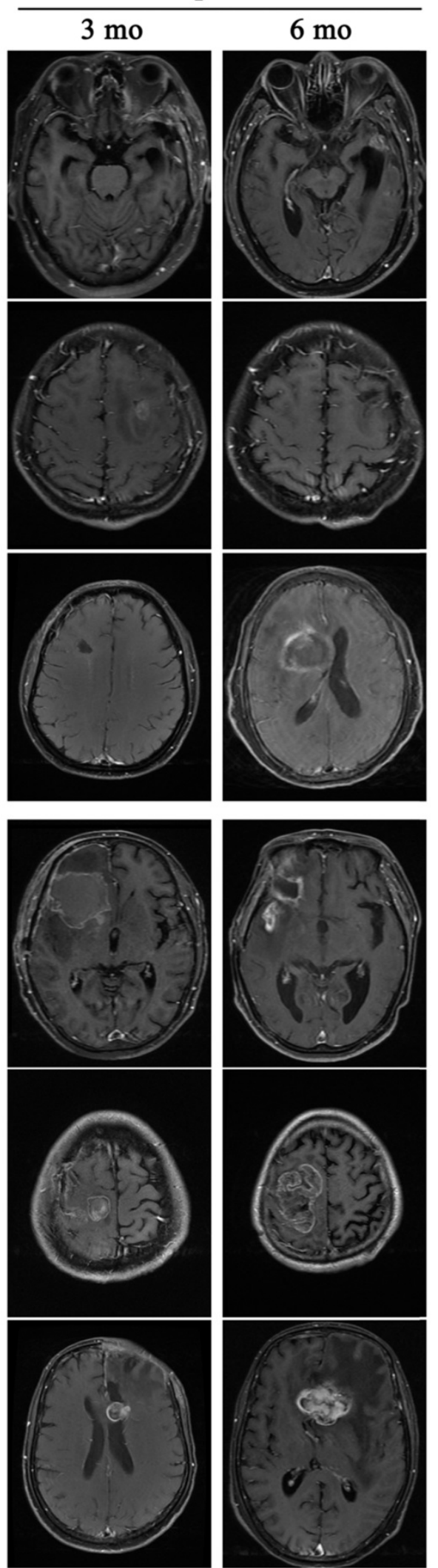

Figure 4. GBM recurrence in patients undergoing PTBE resection is slower than that of PTBE without resection. All the patients are accompanied by peritumoral brain edema before operative. a, patients remove the tumor and give an excision with PTBE, the tumor recurred during the 6 rd month after operative. b, patients only take a resection of the tumor, the tumor recurred during the 3 rd month after operative. 
a

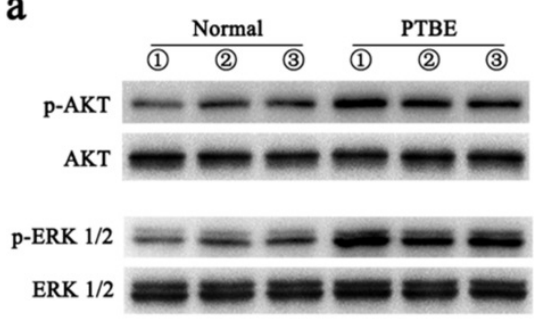

b

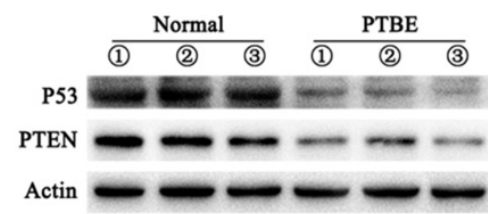

d

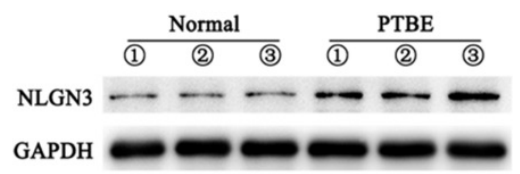

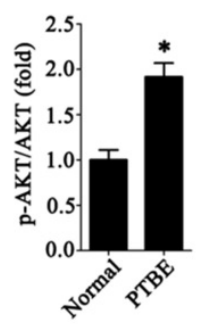

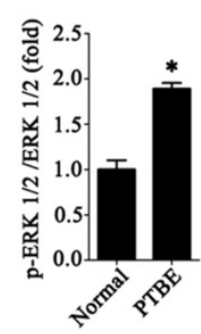

c
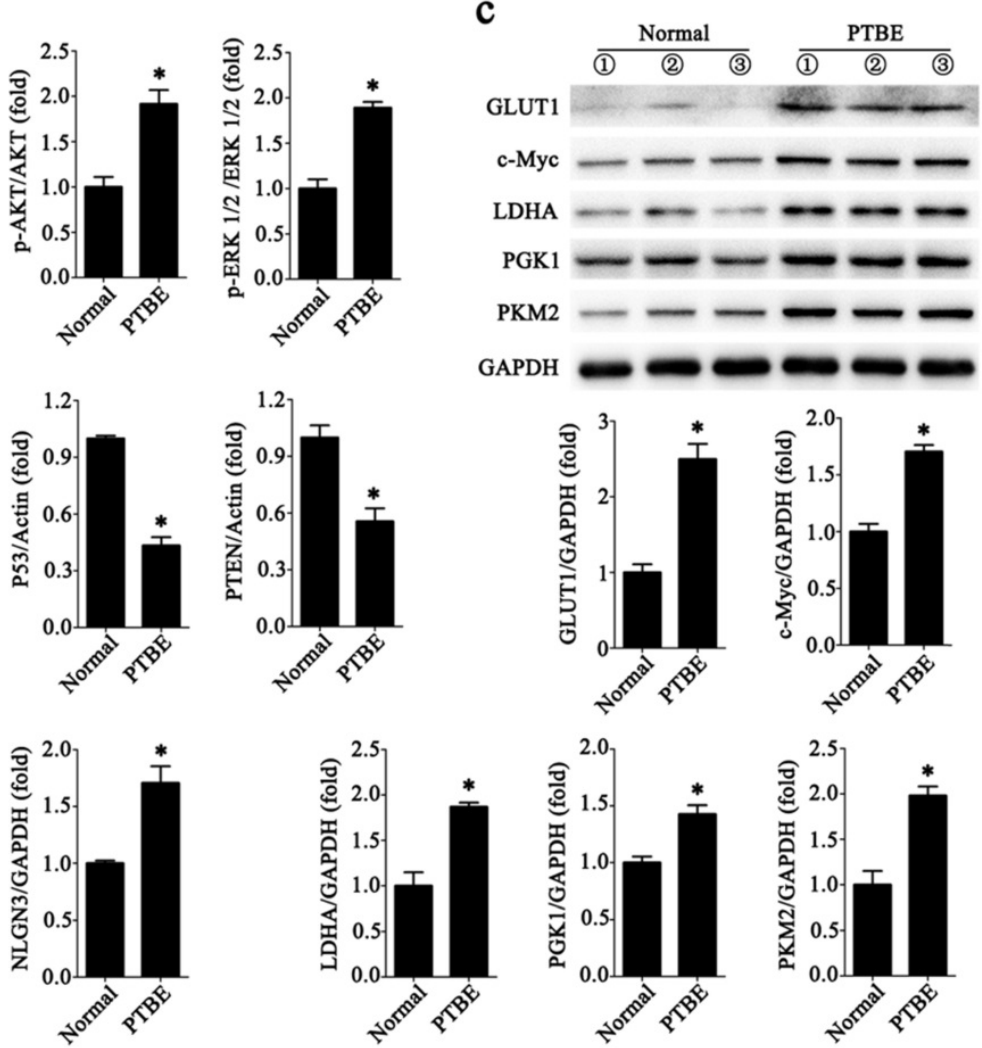

Figure 5. Abnormal expression of tumor-associated proteins in PTBE region. a-d, Western blotting analysis of p-AKT and p-ERK $1 / 2$ (a); P53 and PTEN (b); GLUT1, c-Myc, LDHA, PGK1 and PKM2 (c); NLGN3 (d) in human brain tissues ( $* P<0.05$ versus the Normal). Data are expressed as mean \pm SE. Statistical analysis was implemented by student's t-test and variance analysis.

Currently international guidelines regarding the management of PTBE does not include a formal recommendation to surgically remove PTBE [24]. In clinical practice, surgeons therefore use their clinical judgment to decide whether PTBE tissue should be resected. The standard surgical approach is to maximally resect the tumors while retaining maximum neurological function. We found no significant differences in postoperative complications between patients who received PTBE resection vs those who did not [25]. The KPS scores were also not significantly different in patients with PTBE that was surgically managed compared to non-surgically managed patients [26]. This suggests resection of PTBE tissue has no significant post-operative complications in patients with GBM.

Postoperative recurrence of tumor in patients with GBM is inevitable [27]. In our study, all 207 patients relapsed within 6 months after surgery. However, when comparing recurrence between patients who received surgical resection of PTBE vs those who did not receive surgical treatment, we found that the relapse rate is delayed in the former group. This phenomenon has thus far not been reported in the literature. Interestingly, we also found that certain tumor associated proteins are abnormally expressed in the PTBE region. There was evidence of increased upregulation of AKT, ERK 1/2, PKM2, PGK1, LDHA, c-Myc and GLUT1 in the PTBE region $[13,22,28,29]$. Meanwhile, tumor suppressor gene PTEN and P53 were down-regulated.

Various genes and molecular mediators have been shown to be upregulated in PTBE such as vascular endothelial growth factor (VEGF), aquaporins, Cox-2 and nitric oxide [30]. However, in this study we have highlighted the presence of multiple other genes in PTBE tissue and we therefore speculate whether relapse of GBM is associated with pro-cancerous genes seen in the edematous regions.

\section{Conclusion}

Our findings reveal that GBM is associated with PTBE and when this is surgically treated, can lead to a delay in relapse rates. We found no significant differences in neurological complications or KPS scores between the surgically and non-surgically managed groups. We found that PTBE tissue removal ameliorates the midline shift seen in GBM patients. We also found that postoperative recurrence of GBM patients is delayed in patients who received surgical treatment for PTBE. Most interestingly, we found increased expression of pro-tumor associated genes in 
the PTBE region, which may be associated with the relapse rate. Taken together, we show that GBM-induced PTBE may be a critical factor for postoperative recurrence in GBM patients and thus must be explored further to understand its importance in the pathobiology of GBM and its association with the tumor microenvironment.

\section{Acknowledgements}

This work was supported by the Science Foundation for Youth Scholars of Wuhan University (2042019kf0095), which from Fundamental Research Funds for the central University and China Scholarship Council.

\section{Competing Interests}

The authors have declared that no competing interest exists.

\section{References}

1. Pisapia DJ. The Updated World Health Organization Glioma Classification: Cellular and Molecular Origins of Adult Infiltrating Gliomas. Arch Pathol Lab Med. 2017 Dec; 141(12):1633-45.

2. Woernle CM, Péus D, Hofer S, Rushing EJ, Held U, Bozinov O, et al. Efficacy of Surgery and Further Treatment of Progressive Glioblastoma. World Neurosurg. 2015 Aug: 84(2):301-7.

3. Ideguchi M, Kajiwara K, Goto H, Sugimoto K, Nomura S, Ikeda E, et al. MRI findings and pathological features in early-stage glioblastoma. J Neurooncol. 2015 Jun; 123(2):289-97.

4. Venkatesh HS, Tam LT, Woo PJ, Lennon J, Nagaraja S, Gillespie SM, et al. Targeting neuronal activity-regulated neuroligin-3 dependency in high-grade glioma. Nature. 2017 28; 549(7673):533-7.

5. Liu R, Qin X-P, Zhuang Y, Zhang Y, Liao H-B, Tang J-C, et al. Glioblastoma recurrence correlates with NLGN3 levels. Cancer Med. 2018 May 18;

6. Qin X, Akter F, Qin L, Xie Q, Liao X, Liu R, et al. MicroRNA-26b/PTEN Signaling Pathway Mediates Glycine-Induced Neuroprotection in SAH Injury. Neurochem Res. 2019 Nov; 44(11):2658-69.

7. Cong Z-X, Wang H-D, Wang J-W, Zhou Y, Pan H, Zhang D-D, et al. ERK and PI3K signaling cascades induce Nrf2 activation and regulate cell viability partly through Nrf2 in human glioblastoma cells. Oncol Rep. 2013 Aug; 30(2):715-22.

8. Liu R, Tang J-C, Pan M-X, Zhuang Y, Zhang Y, Liao H-B, et al. ERK $1 / 2$ Activation Mediates the Neuroprotective Effect of $\mathrm{BpV}$ (pic) in Focal Cerebral Ischemia-Reperfusion Injury. Neurochem Res. 2018 Jul; 43(7):1424-38.

9. Milella M, Falcone I, Conciatori F, Cesta Incani U, Del Curatolo A, Inzerilli N, et al. PTEN: Multiple Functions in Human Malignant Tumors. Front Oncol. 2015; 5:24.

10. Huang C-H, Chen Y-T, Lin J-H, Wang H-T. Acrolein induces ribotoxic stress in human cancer cells regardless of p53 status. Toxicol In Vitro. 2018 Oct; 52:26571.

11. McGillen JB, Kelly CJ, Martínez-González A, Martin NK, Gaffney EA, Maini $\mathrm{PK}$, et al. Glucose-lactate metabolic cooperation in cancer: insights from a spatial mathematical model and implications for targeted therapy. J Theor Biol. 2014 Nov 21; 361:190-203.

12. Duan K, Liu Z-J, Hu S-Q, Huo H-Y, Xu Z-R, Ruan J-F, et al. Lactic acid induces lactate transport and glycolysis/OXPHOS interconversion in glioblastoma. Biochem Biophys Res Commun. 2018 05; 503(2):888-94.

13. Yang W, Zheng Y, Xia Y, Ji H, Chen X, Guo F, et al. ERK1/2-dependent phosphorylation and nuclear translocation of PKM2 promotes the Warburg effect. Nat Cell Biol. 2012 Dec; 14(12):1295-304.

14. Zhang Y, Yu G, Chu H, Wang X, Xiong L, Cai G, et al. Macrophage-Associated PGK1 Phosphorylation Promotes Aerobic Glycolysis and Tumorigenesis. Mol Cell. 2018 19; 71(2):201-215.e7.

15. Gupta A, Ajith A, Singh S, Panday RK, Samaiya A, Shukla S, PAK2-c-Myc-PKM2 axis plays an essential role in head and neck oncogenesis via regulating Warburg effect. Cell Death Dis. 2018 01; 9(8):825.

16. Yang W, Lu Z. Nuclear PKM2 regulates the Warburg effect. Cell Cycle. 2013 Oct $1 ; 12(19): 3154-8$.

17. Li X, Jiang Y, Meisenhelder J, Yang W, Hawke DH, Zheng Y, et al. Mitochondria-Translocated PGK1 Functions as a Protein Kinase to Coordinate Glycolysis and the TCA Cycle in Tumorigenesis. Mol Cell. 2016 Mar 3; 61(5):705-19.
18. Schoenegger K, Oberndorfer S, Wuschitz B, Struhal W, Hainfellner J, Prayer D, et al. Peritumoral edema on MRI at initial diagnosis: an independent prognostic factor for glioblastoma? Eur J Neurol. 2009 Jul; 16(7):874-8.

19. Talacchi A, Santini B, Savazzi S, Gerosa M. Cognitive effects of tumour and surgical treatment in glioma patients. J Neurooncol. 2011 Jul; 103(3):541-9.

20. Kim B-W, Kim M-S, Kim S-W, Chang C-H, Kim O-L. Peritumoral brain edema in meningiomas: correlation of radiologic and pathologic features. J Korean Neurosurg Soc. 2011 Jan; 49(1):26-30.

21. Gamburg ES, Regine WF, Patchell RA, Strottmann JM, Mohiuddin M, Young $\mathrm{AB}$. The prognostic significance of midline shift at presentation on survival in patients with glioblastoma multiforme. Int J Radiat Oncol Biol Phys. 2000 Dec 1; 48(5):1359-62.

22. Venkatesh HS, Johung TB, Caretti V, Noll A, Tang Y, Nagaraja S, et al. Neuronal Activity Promotes Glioma Growth through Neuroligin-3 Secretion. Cell. 2015 May 7; 161(4):803-16.

23. Eder K, Kalman B. Molecular heterogeneity of glioblastoma and its clinical relevance. Pathol Oncol Res. 2014 Oct; 20(4):777-87.

24. Da Fonseca CO, Silva JT, Lins IR, Simão M, Arnobio A, Futuro D, et al. Correlation of tumor topography and peritumoral edema of recurrent malignant gliomas with therapeutic response to intranasal administration of perillyl alcohol. Invest New Drugs. 2009 Dec; 27(6):557-64.

25. D'Amico RS, Cloney MB, Sonabend AM, Zacharia B, Nazarian MN, Iwamoto FM, et al. The Safety of Surgery in Elderly Patients with Primary and Recurrent Glioblastoma. World Neurosurg. 2015 Oct; 84(4):913-9.

26. Chambless LB, Kistka HM, Parker SL, Hassam-Malani L, McGirt MJ, Thompson RC. The relative value of postoperative versus preoperative Karnofsky Performance Scale scores as a predictor of survival after surgical resection of glioblastoma multiforme. J Neurooncol. 2015 Jan; 121(2):359-64.

27. Auffinger B, Spencer D, Pytel P, Ahmed AU, Lesniak MS. The role of glioma stem cells in chemotherapy resistance and glioblastoma multiforme recurrence. Expert Rev Neurother. 2015; 15(7):741-52.

28. Yang Y-C, Cheng T-Y, Huang S-M, Su C-Y, Yang P-W, Lee J-M, et al. Cytosolic PKM2 stabilizes mutant EGFR protein expression through regulating HSP90-EGFR association. Oncogene. 2016 30; 35(26):3387-98.

29. Wong N, Ojo D, Yan J, Tang D. PKM2 contributes to cancer metabolism. Cancer Lett. 2015 Jan 28; 356(2 Pt A):184-91.

30. Papadopoulos MC, Saadoun S, Davies DC, Bell BA. Emerging molecular mechanisms of brain tumour oedema. Br J Neurosurg. 2001 Apr; 15(2):101-8. 